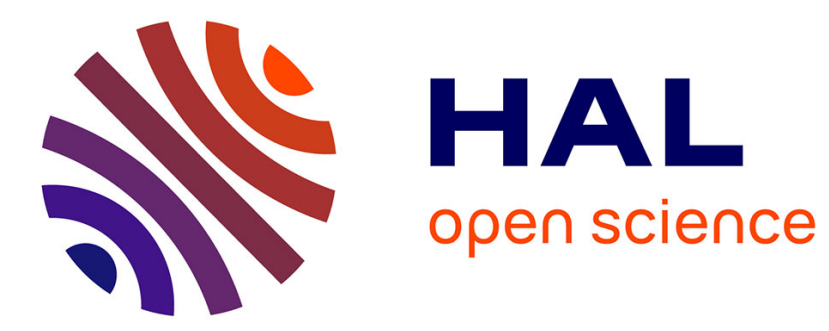

\title{
What can tell the quantum chemical topology on carbon-astatine bonds?
}

Mohamed Amaouch, Gilles F Montavon, Nicolas Galland, Julien Pilmé

\section{To cite this version:}

Mohamed Amaouch, Gilles F Montavon, Nicolas Galland, Julien Pilmé. What can tell the quantum chemical topology on carbon-astatine bonds?. Molecular Physics, 2015, pp.1-8. 10.1080/00268976.2015.1120361 . hal-01258801

\section{HAL Id: hal-01258801 https: / hal.sorbonne-universite.fr/hal-01258801}

Submitted on 19 Jan 2016

HAL is a multi-disciplinary open access archive for the deposit and dissemination of scientific research documents, whether they are published or not. The documents may come from teaching and research institutions in France or abroad, or from public or private research centers.
L'archive ouverte pluridisciplinaire HAL, est destinée au dépôt et à la diffusion de documents scientifiques de niveau recherche, publiés ou non, émanant des établissements d'enseignement et de recherche français ou étrangers, des laboratoires publics ou privés. 


\title{
What Can Tell The Quantum Chemical Topology On Carbon-Astatine \\ Bonds?
}

\author{
Mohamed Amaouch ${ }^{1,2}$, Gilles Montavon ${ }^{3}$, Nicolas Galland ${ }^{4, \dagger}$ and Julien \\ Pilmé ${ }^{1,2, \dagger}$
}

${ }^{1}$ Sorbonne Universités, UPMC Université Paris 06, UMR 7616, Laboratoire de Chimie Théorique, F-75005 Paris, France.

${ }^{2}$ CNRS UMR 7616, Laboratoire de Chimie Théorique, F-75005 Paris, France.

${ }^{3}$ SUBATECH, UMR CNRS 6457, IN2P3/EMN Nantes/Université de Nantes, 4 rue A. Kastler, BP 20722, 44307 Nantes Cedex 3, France.

${ }^{4}$ CEISAM, UMR CNRS 6230, Université de Nantes, 2 Rue de la Houssinière, BP 92208,44322 Nantes Cedex 3, France.

CorrespondingAuthors:

† Email: pilme@1ct.jussieu.fr

† Email: nicolas.galland@ univ-nantes.fr

\begin{abstract}
The nature of carbon-astatine bonds involved in some model species that mimic ${ }^{211}$ At-labelled biomolecules, was investigated by means of ELF and QTAIM analyzes in a context of two-component relativistic computations. The nature of the bonded carbon atom proved to be decisive. When At is bonded to an ethynyl group, some charge delocalization with the vicinal triple $\mathrm{C}-\mathrm{C}$ bond strengthens the At- $\mathrm{C}$ bond and gives it a multiple bond character. However, At displays also a large positive charge which may alter the in vivo stability of such At-C bonds. In the case of an isopropyl group, the At-C bond is less polarized but also much weaker. In contrast, the bond remains strong whilst retaining a small At positive charge when At is bonded to an $\mathrm{sp}^{2}$ carbon atom. Hence, these latter results rationalize why aromatic or aryl groups appear reasonably suited for a priori stable radiolabelling of biomolecules with ${ }^{211}$ At in the context of alpha therapy.
\end{abstract}

Keywords: QTAIM. ELF. Topological Analysis. Quantum Chemical Topology. Relativistic effects. Astatine. Radiolabelling. 


\section{Introduction}

Understanding the nature of the chemical bonding in systems containing heavy elements is not only of fundamental interest, but it is also crucial for applications of societal interest (e.g. nuclear medicine) or environmental questions (e.g. nuclear waste management). However, this purpose can be hampered by relativistic effects and in particular the spin-orbit coupling (SOC). Indeed, the latter can complicate a lot the reading of bonding schemes when heavy elements are involved. One may distinguish the scalar relativistic effects (spin-independent) from the spin-dependent ones, and especially the coupling between the electron spin momentum and the orbital momentum. SOC requires to expand the wave functions using spinors. The spinors can mix different symmetries ( $\sigma$ and $\pi$ ) and, as they are complex vector functions, they do not lend easily to visualization [1-3]. Because the spinors are generally not well suited for the bonding analysis in complex systems, one may wish to use a strategy based on a physical partition of the geometrical space rather than an analysis of spinor properties. The quantum chemical topology (QCT) appears as a powerful tool to scrutinize bonds breaking and formation, as the QCT yields some representations that can be easily related to usual objects for chemists: atoms, bonds, lone-pairs, etc. This methodology aims at answering questions about the nature and the properties of chemical bonds, reactivity and chemical reactions. QCT relies on the gradient field analysis of onedensity functions, just as the Quantum Theory of Atoms In Molecules (QTAIM) [4] and the topological analysis of the Electron Localization Function (ELF) [5], which have been widely reviewed in numerous articles and books [6-10]. Recently, some of us have introduced the ELF and the QTAIM analyzes in the field of two-component (2c) relativistic quantum calculations in order to uncover SOC effects on the bonding scheme of molecular systems containing heavy elements [11, 12]. Among these systems, some contain the astatine radioelement (At, $Z=85$ ) which is known for displaying spectacular SOC effects [11]. At is the heaviest naturally occurring halogen and it has focused attention because one of its longest-lived radioisotopes, ${ }^{211} \mathrm{At}$, is very promising for alpha-radioimmunotherapy [13]. However, injected free At in human body may damage healthy tissues and the general approach is to use ${ }^{211}$ At-labelled biomolecules (amino acids, proteins, antibodies, etc), which have a high affinity for the targeted cancer cells. The study of the At-C interaction is of great interest since most of the currently developed ${ }^{211}$ At-labelling protocols involve the formation of an At-C bond. For instance, Figure 1 displays labelled cholesterol and steroid derivatives that 
were synthesized for potential use in targeted radiotherapy as they can be distributed and localized in diseased areas very rapidly [14-16]. In order to limit the deastatination in vivo of the labelled biomolecules, which results in the release of free astatine, one argument raised in the literature for improving the stability of the At-bonds is to bind At to aromatic systems rather than to vinyl or alkyl carbons [13]. One may expect an increase of the At-C bond multiplicity

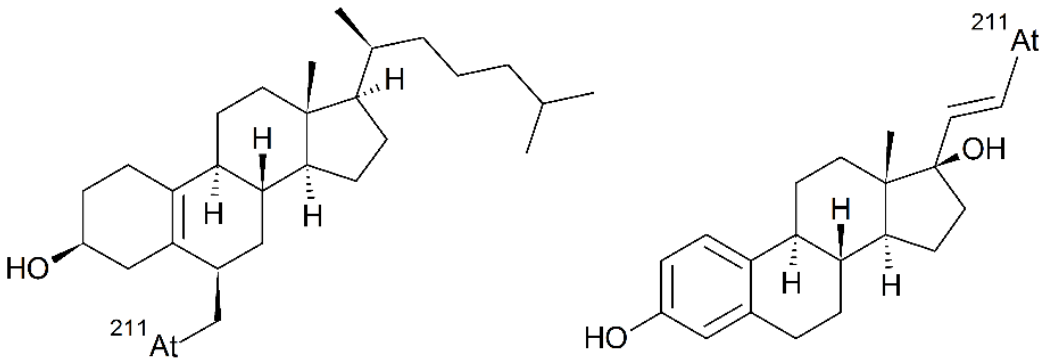<smiles>COc1ccc(CC(N)C(=O)O)cc1[Al]</smiles>

Figure 1. Structures of 6-astatomethyl-19-norcholest-5(10)-en-3 $\beta$-ol (left), 17-astatovinyl-estra1,3,5(10)-triene-3,17 $\alpha$-diol (middle), and 3-astato-4methoxyphenylalanine (right).

Thus, we focused in this paper on the nature of At-C interactions in four model Atspecies that mimic ${ }^{211}$ At-labelled biomolecules. As shown in Scheme 1, these species have been selected according to the hybridization state of the carbon atom bonded to astatine, namely At- $\mathrm{CH}\left(\mathrm{CH}_{3}\right)_{2}$ (At-isopropyl), At- $\mathrm{C}_{6} \mathrm{H}_{5}$ (At-phenyl), At- $\mathrm{CH}=\mathrm{CH}_{2}$ (Atvinyl) and At-C $\equiv \mathrm{CH}$ (At-ethynyl). If the covalent character of the $\mathrm{C}_{1}-\mathrm{C}_{2}$ bond is indeed expected to increase from a single bond (At-isopropyl) to a triple bond (At-ethnyl), the properties of the At- $\mathrm{C}_{1}$ bond remain to be established, in particular if the density delocalization varies between astatine and its substituent. Although the selected Atspecies are models, some disclosed features may remain and can help to rationalize the results of in vivo experiments with ${ }^{211}$ At-labelled biomolecules.

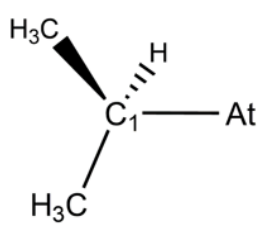

At-isopropyl

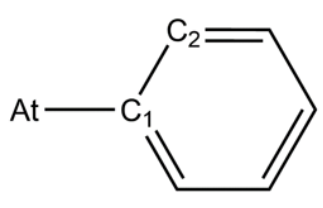

At-phenyl

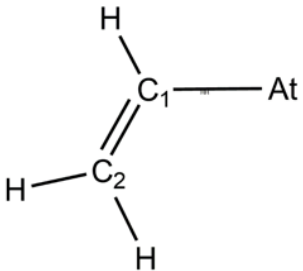

At-vinyl

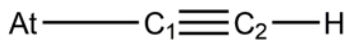

At-ethynyl

Scheme 1: Highlighted carbon-astatine bonds in the studied species. 


\section{Theory}

\section{a. Savin's interpretation of ELF}

In 1990, Becke and Edgecombe designed the ELF function in order to identify "localized electronic groups in atomic and molecular systems"[17]. They have proposed to examine the $D_{\sigma}(\mathbf{r})$ quantity arising from the Taylor series expansion of the spherical average conditional same-spin pair density. Indeed, $D_{\sigma}(\mathbf{r})$ measures the short-range behavior of an electron with $\sigma$ spin approaching the reference electron of same $\sigma$ spin, and appears as a signature of the electron-pair distribution. The authors propose to scale $\mathrm{D}_{\sigma}(\mathbf{r})$ by the homogeneous electron gas kinetic energy density $\mathrm{D}^{0}{ }_{\sigma}(\mathbf{r})=2.871 \rho_{\sigma}(\mathrm{r})^{5 / 3}$, which is then used as a reference. In addition, they propose to confine the ratio $\mathrm{D}_{\sigma}(\mathbf{r}) / \mathrm{D}^{0}{ }_{\sigma}(\mathbf{r})$ in the $[1 ; 0]$ interval with a Lorentzian form such as $E L F(\mathbf{r})=$ $\left[1+\left(\frac{D_{\sigma}(r)}{D_{\sigma}^{0}(r)}\right)^{2}\right]^{-1}$. Hence, ELF tends to 1 in regions of the physical space where finding electrons with parallel spins is highly improbable. The function is independent of any unitary transformation of the orbitals, and, in principle, it is derivable from the electron density. Thereafter, different physical interpretations were proposed for ELF but the one of Andreas Savin [18, 19] deserves a special attention. Beyond conditional pair densities interpretations, $D_{\sigma}(r)$ can be generalized to any $\rho(\mathbf{r})$ density independently of the spin and the obtained formulation was:

$$
\mathrm{D}(\mathbf{r})=\frac{1}{2} \sum_{\mathrm{i}}^{\mathrm{occ}}\left|\nabla \varphi_{\mathrm{i}}\right|^{2}-\frac{|\nabla \rho(\mathbf{r})|^{2}}{8 \rho(\mathbf{r})}=\mathrm{T}_{\mathrm{s}}(\mathbf{r})-\mathrm{T}_{\mathrm{w}}(\mathbf{r})
$$

Where $T_{s}(\mathrm{r})$ is the definite positive kinetic energy density, built from the molecular orbitals $\varphi_{\mathrm{i}}$, and $T_{w}(\mathbf{r})$ is the von Weizsäcker kinetic energy density, i.e. the kinetic energy of a density model system in which the antisymmetry is switched off [20]. Hence, $\mathrm{D}(\mathbf{r})$ can be understood as a measure of the excess kinetic energy due to the Pauli exclusion principle. This interpretation appeared as a cornerstone of the concept of electron-pairs localization in the ELF framework. It is easy to verify in closed-shell systems that $\mathrm{D}(\mathbf{r})=\mathrm{D}_{\sigma}(\mathbf{r})$. The final ELF formulation is then written as follows: 


$$
\operatorname{ELF}(r)=\left[1+\left(\frac{\mathrm{T}_{\mathrm{s}}(\mathrm{r})-\mathrm{T}_{\mathrm{w}}(\mathrm{r})}{\mathrm{D}^{0}(\mathbf{r})}\right)^{2}\right]^{-1}
$$

Note that beyond the DFT framework, the calculation of ELF has been recently extended to $a b$ initio correlated wave functions [21].

\section{b. ELF from 2c-spinors}

In a quasirelativistic framework, the nonrelativistic formalism operating with orbitals is extended to a $2 \mathrm{c}$ formalism where the wave function is built from singleparticle functions known as (pseudo-) spinors, $\varphi_{i}(\mathbf{r})$. The latter are no longer of pure $\alpha$ or $\beta$ character, but have both an $\alpha$ and a $\beta$ complex component (hence, two components). Usually, $\varphi_{i}(\mathbf{r})$ are expanded using atom-centered gaussian basis functions, $\chi_{\mu}(\mathbf{r})$, and the expansion coefficients, $c_{i}^{\alpha}$ and $c_{i}^{\beta}$, are complex and determined within the SCF procedure:

$$
\varphi_{i}(\mathbf{r})=\left(\begin{array}{l}
\varphi_{i \alpha}(\mathbf{r}) \\
\varphi_{i \beta}(\mathbf{r})
\end{array}\right)=\left(\begin{array}{l}
\sum_{\mu} c_{i \mu}^{\alpha} \chi_{\mu}(\mathbf{r}) \\
\sum_{\mu} c_{i \mu}^{\beta} \chi_{\mu}(\mathbf{r})
\end{array}\right)
$$

The electron density is then defined as:

$$
\begin{array}{r}
\rho(\mathbf{r})=\sum_{\mathrm{i}}^{\mathrm{occ}} \varphi_{\mathrm{i}}^{\dagger}(\mathbf{r}) \varphi_{\mathrm{i}}(\mathbf{r})=\sum_{\mathrm{i}}^{\mathrm{occ}}\left(\varphi_{\mathrm{i} \alpha}^{*}(\mathbf{r}) \varphi_{\mathrm{i} \alpha}(\mathbf{r})+\varphi_{\mathrm{i} \beta}^{*}(\mathbf{r}) \varphi_{\mathrm{i} \beta}(\mathbf{r})\right) \\
=\sum_{\mu} \sum_{\nu} \mathrm{P}_{\mu \nu} \chi_{\mu}(\mathbf{r}) \chi_{\nu}(\mathbf{r})
\end{array}
$$

Where $P_{\mu \nu}$ is the total density matrix element defined from the expansion coefficients $c_{i}^{\alpha}$ and $c_{i}^{\beta}$ :

$$
\mathrm{P}_{\mu \nu}=\sum_{\mathrm{i}}^{\mathrm{occ}}\left(\mathrm{c}_{\mathrm{i} \mu}^{\alpha}\left(\mathrm{c}_{\mathrm{iv}}^{\alpha}\right)^{*}+\mathrm{c}_{\mathrm{i \mu}}^{\beta}\left(\mathrm{c}_{\mathrm{iv}}^{\beta}\right)^{*}\right)
$$


Note that $P_{\mu \nu}$ elements are complex for each component, $\alpha$ and $\beta$. Beyond this density formulation, some of us have recently proposed to extend the ELF formulation (eq. 2) to the 2c-DFT relativistic formalism $[11,12]$. This formulation can readily be calculated from occupied $2 \mathrm{c}$ spinors and is safe for practical use on closed-shell species where the spin polarization is small (Kramers-restricted closed-shell approximation). The different quantities appearing in ELF are evaluated from spinors and $P_{\mu \nu}$ elements of the total electron density (eq. 5).

\section{c. Topological analysis}

This methodology lies on the analysis of the gradient field of a scalar function by applying the theory of dynamical systems $[22,23]$. The latter provides a partitioning of the molecular space into non-overlapping volumes (basins, labeled $\Omega$ ). The basins are then localized around maxima of the function and are separated by zero flux surfaces. In the QTAIM theory [7], the function of interest is the electron density. Each atom of a molecule corresponds to a basin and the integration of the electron density over the basin volume provides an atomic population. The atomic charge of a topological atom $\Omega, q(\Omega)$, is calculated by subtracting the atomic population from the atomic number, $Z(\Omega)$. If effective core potentials (ECPs) are used for some atoms, the calculation of their atomic charge involves $Z_{\text {eff }}$, the charge of the inner-core. Introduced by Bader and Stephens [24], and later recovered by Fradera et al. [9], the delocalization index (DI) is a measure of the electron-sharing between two atoms and can be compared to other bond order indices. Moreover, the critical points of the scalar function and their connectivity provide a characterization of the topology of the gradient field. The bond critical point (BCP) plays a central role [25]. Indeed, some local indicators calculated at the $\mathrm{BCP}$ provide information on the nature of the interactions between atoms. For example, the electron density at the BCP, $\rho_{\mathrm{b}}$, is in general (i) larger than $0.20 \mathrm{e} \mathrm{bohr}^{-3}$ in shared-shell interactions, in other words, covalent bonds, and (ii) smaller than $0.10 \mathrm{e} \mathrm{bohr}^{-3}$ in closed-shell interactions, e.g. ionic, van der Waals or hydrogen bonding [26]. For shared-shell interactions, the Laplacian of the density at the BCP, $\nabla^{2} \rho_{b}$, is negative, showing a local concentration of charge at the BCP. In addition, the ratio $\left|V_{\mathrm{b}}\right| G_{\mathrm{b}}\left(V_{\mathrm{b}}\right.$ and $G_{\mathrm{b}}$ being, respectively, the potential energy density and the kinetic energy density at the $\mathrm{BCP}$ ) reflects the covalency magnitude of the chemical bond [27]. 
In 1994, B. Silvi and A. Savin proposed the topological analysis of ELF [5]. Briefly, the topology of ELF exhibits atomic and non-atomic maxima that can be related to chemical concepts issued from the Lewis theory. The core basins (if $Z>2$ ) are located at nuclear centers whereas the valence basins are located in the remaining space. These valence basins are characterized by a synaptic order, i.e. the number of core basins with which they share a common boundary [28]. Monosynaptic basins, labeled $\mathrm{V}(\mathrm{X})$, usually correspond to the lone-pair regions of $\mathrm{X}$ atoms while disynaptic basins, labeled $\mathrm{V}(\mathrm{X}, \mathrm{Y})$, correspond to two-center $\mathrm{X}-\mathrm{Y}$ bonds. The integration of the electron density over the basin volume provides a covalent bond population for $\mathrm{V}(\mathrm{X}, \mathrm{Y})$ or a lone-pair population for $\mathrm{V}(\mathrm{X})$. Beyond the population analysis, the ELF local moments indicate local polarizations of valence basins immersed in a given chemical environment [29]. These moments give access to the local polarization of the lone-pairs and bonds. The magnitude of the first moments $|\mathrm{M} 1|$ give access to the basin dipolar polarizations, while the magnitude of second moments $|\mathrm{M} 2|$ provide quadrupolar polarizations. It has been shown that the dipolar polarization is related to the chemical reactivity and the bond quadrupolar polarizations are related to the bonds $\pi$ character. Hence, |M2| enables the sorting of families of molecules according to their bond orders. The combination of ELF and QTAIM topologies has led Raub and Jansen [30] to propose a bond polarity index $p_{X Y}$ designed for a disynaptic basin $\mathrm{V}(\mathrm{X}, \mathrm{Y})$ :

$$
p_{X Y}=\frac{\overline{\mathrm{N}}[V(X, Y) \mid X]-\overline{\mathrm{N}}[V(X, Y) \mid Y]}{\overline{\mathrm{N}}[V(X, Y)]}
$$

Where $\overline{\mathrm{N}}[V(X, Y) \mid X]$ and $\overline{\mathrm{N}}[V(X, Y) \mid Y]$ give the contributions of $\mathrm{X}$ and Y QTAIM basins, respectively, to the $\overline{\mathrm{N}}[V(X, Y)]$ total population. By definition, a strongly polarized bond yields an index close to 1 .

\section{Computational details}

Geometry optimizations were performed using the quasirelativistic spin-orbit DFT method (SODFT), implemented in the NWChem program package [31]. Harmonic vibrational frequencies were evaluated by two sided finite differences of analytical gradients. The B3LYP hybrid functional was used for all calculations [32], since the performance of B3LYP, used in the framework of SODFT calculations, was thoroughly assessed for At-species in several previous works [11, 33-35]. The ECP60MDF pseudopotential was used to mimic the role of the 60 core electrons of At [36], while for the 
remaining electrons, the dhf-TZVPD-2c basis set was used [37, 38]. For the lighter atoms, $\mathrm{H}$ and $\mathrm{C}$, we used the def2-TZVPD basis sets [38]. Note that the form of the pseudo-potential (PP) used in this work is:

$$
\widehat{\mathrm{V}}_{\mathrm{PP}}(\mathbf{r})=-\frac{Z_{\mathrm{eff}}}{\mathbf{r}}+\sum_{\mathrm{ljk}}\left(\mathrm{B}_{\mathrm{lj}}^{\mathrm{k}} e^{-\beta_{\mathrm{lj}}^{\mathrm{k}} \mathrm{r}^{2}} \widehat{\mathrm{P}}_{\mathrm{lj}}\right)
$$

where $Z_{\text {eff }}$ is the charge of the inner-core. The sum runs over a gaussian expansion (index $k$ ) of semi-local short-range radial potentials which are different for different orbital angular-momentum quantum numbers $l$ and, for a given $l$, for the two total oneelectron angular-momentum quantum numbers $j=l \pm 1 / 2\left(\hat{P}_{l j}\right.$ is the 2 c projector onto the complete space of functions with angular symmetry $l, j$ around the core under study). The parameters $B_{l j}^{k}$ and $\beta_{l j}^{k}$ are adjusted so that $\widehat{V}_{P P}$ in 2c valence-only atomic calculations reproduces, as closely as possible, a set of all-electron multiconfiguration Dirac-Hartree-Fock (MCDHF) energies. For ECP60MDF, a transcription of the PP into a scalar-relativistic spin-averaged part and an effective one-electron spin-orbit operator $\left(\widehat{V}_{S O}\right)$ was made [36]. The action of $\widehat{V}_{S O}$ generates a wave function built of singleparticle functions known as pseudo-spinors. The omission of $\widehat{V}_{S O}$ in the calculation leads to one-component (1c) scalar-relativistic approach. Hence, SOC effects can readily be quantified via the difference between SODFT calculations, with $\widehat{V}_{S O}$ included in the PP, and DFT calculations, without $\widehat{V}_{S O}$ included in the PP. In order to evaluate SOC effects on studied species, geometry optimizations (and frequency calculations) have been also done at scalar-relativistic level through DFT calculations. Afterward, new QTAIM and ELF topological analyses have been carried out. Note that for all computations, the At-C bond of the studied species was aligned with the $z$-axis.

The QTAIM and ELF topological analyses, extended for the treatment of $2 \mathrm{c}$ wave functions from the NWChem program package, are implemented in a modified version of the TopMod program package [11, 39]. ELF isosurfaces have been drawn using the Molekel software [40].

\section{Results and Discussion}

The performances of the 2c-B3LYP/dhf-TZVPD-2c calculations regarding the studied species can be checked using the Table 1 . In the case of the At- $\mathrm{CH}\left(\mathrm{CH}_{3}\right)_{2}$ and 
At- $\mathrm{C}_{6} \mathrm{H}_{5}$ species, the At- $\mathrm{C}_{1}$ bond enthalpies are calculated in good agreement with the available experimental dissociation energies [41]. The corresponding values show an increase about $10 \mathrm{kcal} \mathrm{mol}^{-1}$ if At is bonded to an $\mathrm{sp}^{2}$ carbon atom instead of an $\mathrm{sp}^{3}$ one. Furthermore, the At- $\mathrm{C}_{1}$ bond is predicted for the At-C $\equiv \mathrm{CH}$ species at least $20 \mathrm{kcal} \mathrm{mol}^{-1}$ stronger than for At- $\mathrm{CH}=\mathrm{CH}_{2}$ and At- $\mathrm{C}_{6} \mathrm{H}_{5}$.

Table 1. Computed At- $\mathrm{C}_{\mathbf{1}}$ bond enthalpies and selected bond distances at the $2 \mathrm{c}$ -

B3LYP/dhf-TZVPD-2c level of theory.

\begin{tabular}{c||c||c||c|c}
\hline \multicolumn{1}{c||}{} & \multicolumn{2}{c||}{$\begin{array}{c}\Delta H^{\circ}{ }_{298} \\
\left(\mathrm{kcal} \mathrm{mol}^{-1}\right)\end{array}$} & \multicolumn{2}{c}{$\begin{array}{c}\text { Bond distances } \\
(\AA)\end{array}$} \\
\hline \hline System & calc. & exp. $^{a}$ & $R_{A t-C l}$ & $R_{\text {Cl-C2 }}$ \\
\hline \hline At-isopropyl & 31.5 & $36.3 \pm 2.3$ & 2.357 & 1.513 \\
At-vinyl & 43.1 & & 2.244 & 1.319 \\
At-phenyl & 43.8 & $44.9 \pm 5.1$ & 2.257 & 1.389 \\
At-ethynyl & 66.4 & & 2.141 & 1.204 \\
\hline \hline
\end{tabular}

${ }^{a}$ Experimental dissociation energies from ref [41].

Figure 2 displays the ELF localization domains of all studied species. In addition to core basins, the ELF topology yields several valence basins. Among them, the V(At) basins account for At lone-pairs and the $\mathrm{V}\left(\mathrm{At}, \mathrm{C}_{1}\right)$ ones testify to the covalent bonding between the At and $\mathrm{C}_{1}$ atoms. As expected, disynaptic $\mathrm{V}\left(\mathrm{C}_{1}, \mathrm{C}_{2}\right)$ basins are also found for the $\mathrm{C}-\mathrm{C}$ covalent bonds. 


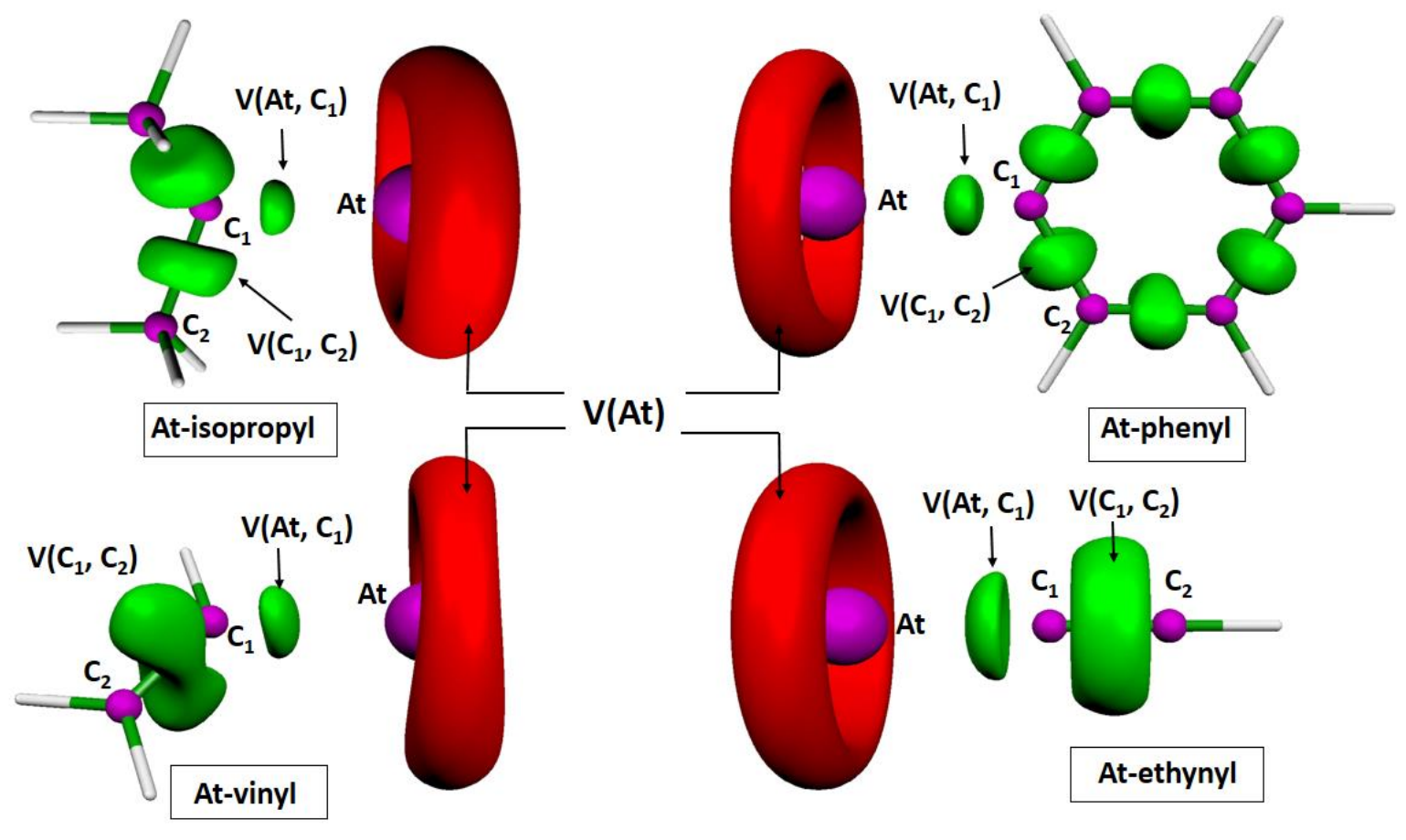

Figure 2. ELF localization domains obtained at the 2c-B3LYP/dhf-TZVPD-2c level of theory for the studied species $(E L F=0.78)$. Color code. magenta: cores $C(A t)$ and $C(C)$, red: monosynaptic basin $\mathrm{V}(\mathrm{At})$, green: disynaptic basins $\mathrm{V}\left(\mathrm{At}, \mathrm{C}_{1}\right)$ and $\mathrm{V}(\mathrm{C}, \mathrm{C})$. The protonated basins, $\mathrm{V}(\mathrm{C}, \mathrm{H})$, have been omitted for clarity.

The integrated ELF properties are presented in Table 2.

Table 2. ELF population analysis at the 2c-B3LYP/dhf-TZVPD-2c level of theory.

\begin{tabular}{l||c||cccc||cc}
\hline \hline & \multicolumn{1}{|c|}{$C(A t)+V(A t)$} & \multicolumn{3}{|c||}{$V\left(A t, C_{1}\right)$} & \multicolumn{2}{|c}{$V\left(C_{1}, C_{2}\right)$} \\
\hline \hline \multicolumn{1}{c|}{ System } & $\bar{N}(\mathrm{e})$ & $\bar{N}(\mathrm{e})$ & $p_{A t C l}$ & $\left|M_{2}\right|\left(\mathrm{e} \mathrm{bohr}^{2}\right)$ & $\pi^{\mathrm{c}}$ & $\bar{N}(\mathrm{e})$ & $\left|M_{2}\right|\left(\mathrm{e} . \mathrm{bohr}^{2}\right)$ \\
\hline \hline At-isopropyl & 24.52 & 1.26 & 0.20 & 0.04 & 0.22 & 1.94 & 0.39 \\
At-vinyl & 24.38 & 1.64 & 0.38 & 0.40 & 0.36 & $3.32^{\mathrm{a}}$ & $3.37^{\mathrm{a}}$ \\
At-phenyl & 24.36 & 1.68 & 0.39 & 0.41 & 0.36 & 2.78 & 2.53 \\
At-ethynyl & 24.14 & 2.25 & 0.59 & 0.66 & 0.70 & 5.02 & $\mathrm{~b}$ \\
\hline \hline
\end{tabular}

a The two basins $\mathrm{V}\left(\mathrm{C}_{1}, \mathrm{C}_{2}\right)$ have been merged into a superbasin (see Figure 2).

${ }^{\mathrm{b}}$ Degenerated attractor.

${ }^{\mathrm{c}}$ The $\pi$ population (e) of the V(At, $\mathrm{C}_{1}$ ) basins is evaluated by only taking into account the expansion coefficients of the $p_{\mathrm{x}}$ and $p_{\mathrm{y}}$ Gaussian basis functions during the integration of the electron density over the basin volumes. 
The population of the $\mathrm{V}\left(\mathrm{C}_{1}, \mathrm{C}_{2}\right)$ basins varies in good agreement with the formal bond order of the $\mathrm{C}_{1}-\mathrm{C}_{2}$ bond: 1.94 e for At- $\mathrm{CH}\left(\mathrm{CH}_{3}\right)_{2}$, where 2 electrons are expected for purely covalent single bond; 2.78 e for At $-\mathrm{C}_{6} \mathrm{H}_{5}$, where one may expect something between a single and a double bond; 3.32 e for At- $\mathrm{CH}=\mathrm{CH}_{2}$, which approximates the 4 electrons of a perfect double bond; 5.02 e for At-C $\equiv \mathrm{CH}$, which supports a higher bond order close to 3 . Accordingly, the $\mathrm{C}_{1}-\mathrm{C}_{2}$ bond distances increase in the reverse order (see Table 1). As shown in Table 3, the features of the QTAIM descriptors at the BCPs of all $\mathrm{C}_{1}-\mathrm{C}_{2}$ bonds strengthen the ELF results.

Table 3. QTAIM analysis at the 2c-B3LYP/dhf-TZVPD-2c level of theory.

\begin{tabular}{lc||cccc||ccc}
\hline \hline & & \multicolumn{5}{|c||}{$A t-C_{l}$} & \multicolumn{3}{c}{$C_{I^{-}} C_{2}$} \\
\hline \hline System & $q(A t)$ & $\rho_{b}\left(\mathrm{e} \mathrm{bohr}^{-3}\right)$ & $\nabla^{2} \rho_{b}\left(\mathrm{e} \mathrm{bohr}^{-5}\right)$ & $\left|V_{b}\right| / G_{b}$ & $\mathrm{DI}(\mathrm{e})^{\mathrm{a}}$ & $\rho_{b}\left(\mathrm{e} \mathrm{bohr}{ }^{-3}\right)$ & $V^{2} \rho_{b}\left(\mathrm{e} \mathrm{bohr}^{-5}\right)$ & $\mathrm{DI}(\mathrm{e})^{\mathrm{a}}$ \\
\hline \hline At-isopropyl & 0.01 & 0.087 & 0.013 & 1.90 & 1.02 & 0.255 & -0.659 & 1.03 \\
At-vinyl & 0.13 & 0.105 & 0.026 & 1.87 & 1.14 & 0.361 & -1.139 & 1.90 \\
At-phenyl & 0.15 & 0.104 & 0.022 & 1.88 & 1.13 & 0.321 & -0.954 & 1.38 \\
At-ethynyl & 0.40 & 0.120 & 0.094 & 1.70 & 1.26 & 0.419 & -1.234 & 2.74 \\
\hline \hline
\end{tabular}

${ }^{\mathrm{a}}$ Delocalization Index, calculated at the scalar-relativistic B3LYP level of theory, provides the intra- and interatomic distribution of electron pairs.

These descriptors are indeed associated with shared-shell interactions, $\rho_{\mathrm{b}}$ being notably large $\left(>0.26 \mathrm{e} \mathrm{bohr}^{-3}\right)$ and $\nabla^{2} \rho_{\mathrm{b}}$ always negative $\left(<-0.66 \mathrm{e} \mathrm{bohr} r^{-5}\right)$. Furthermore, the DI provides a clear ranking of the covalent $\mathrm{C}_{1}-\mathrm{C}_{2}$ bonds since the calculated values agree well with the expected formal bond orders (1, 1.5, 2 and 3), and correlate closely with the increase of the ELF bonding populations.

The trend regarding the population of the $\mathrm{V}\left(\mathrm{At}, \mathrm{C}_{1}\right)$ basins is less clear cut. At first, a rather depleted population of 1.26 e is found for At- $\mathrm{CH}\left(\mathrm{CH}_{3}\right)_{2}$, which deviates from the expected value of 2 electrons for a purely covalent single bond. When At is bonded to a $\mathrm{sp}^{2}$ carbon atom, the $\mathrm{V}\left(\mathrm{At}, \mathrm{C}_{1}\right)$ population increases to $1.64 \mathrm{e}$ and $1.68 \mathrm{e}$, in the case of At- $\mathrm{CH}=\mathrm{CH}_{2}$ and At- $\mathrm{C}_{6} \mathrm{H}_{5}$ species, respectively. In contrast, a population of $2.25 \mathrm{e}$ is found for At- $\mathrm{C} \equiv \mathrm{CH}$. This value suggests a non-negligible multiple bond character owing to a charge delocalization with the vicinal triple $\mathrm{C}-\mathrm{C}$ bond. The $|\mathrm{M} 2|$ values and the $\pi$ contributions to $\mathrm{V}\left(\mathrm{At}, \mathrm{C}_{1}\right.$ ) basins support this analysis (see Table 2). 
Indeed, the $|\mathrm{M} 2|$ value is markedly increased in At- $\mathrm{CH}=\mathrm{CH}_{2}$ and $\mathrm{At}-\mathrm{C}_{6} \mathrm{H}_{5}$ species ( $\sim 0.40$ a.u.) with respect to At- $\mathrm{CH}\left(\mathrm{CH}_{3}\right)_{2}\left(0.04\right.$ a.u.), indicating that the At- $\mathrm{C}_{1}$ bonds of the former species acquire some $\pi$ character. Furthermore, the electron population of the $\mathrm{V}\left(\mathrm{At}, \mathrm{C}_{1}\right)$ basin, described by the $p$ Gaussian basis functions that are perpendicular to the At- $\mathrm{C}_{1}$ bond, is almost two times larger for the At-CH=CH and At- $\mathrm{C}_{6} \mathrm{H}_{5}$ species $(0.36 \mathrm{e})$ than for At- $\mathrm{CH}\left(\mathrm{CH}_{3}\right)_{2}(0.22 \mathrm{e})$. In the case of At- $\mathrm{C} \equiv \mathrm{CH}$, the $|\mathrm{M} 2|$ value and the $\pi$ population of $\mathrm{V}\left(\mathrm{At}, \mathrm{C}_{1}\right)$ are even greater $(0.66$ a.u. and $0.70 \mathrm{e}$, respectively). This analysis is fully consistent with the bond orders provided by the DI: about 1.13 for the At- $\mathrm{CH}=\mathrm{CH}_{2}$ and At- $\mathrm{C}_{6} \mathrm{H}_{5}$ species, and 1.26 for At-C $\equiv \mathrm{CH}$ (see Table 3). Note that the growth of the At- $\mathrm{C}_{1}$ bond multiplicity, from the At- $\mathrm{CH}\left(\mathrm{CH}_{3}\right)_{2}$ species to the At-C $\equiv \mathrm{CH}$ one, comes with the regular shortening of this bond as well as its strengthening (see Table 1). The At- $\mathrm{C}_{1}$ bond strengthening should also be related to the regular increase of the $\sigma$ bonding population, given that the $\sigma$ population increase in $\mathrm{V}\left(\mathrm{At}, \mathrm{C}_{1}\right)$ basins is similar to the one of the $\pi$ population.

The covalent character of the At- $\mathrm{C}_{1}$ bond in the four studied species is also disclosed by the QTAIM descriptors (see Table 3). Indeed, the associated $\left|\mathrm{V}_{\mathrm{b}}\right| \mathrm{G}_{\mathrm{b}}$ ratio is always larger than 1 , which discloses some covalency. However, $\rho_{\mathrm{b}}$ at the $\mathrm{BCP}$ of the At- $\mathrm{C}_{1}$ bond is notably weak $\left(<0.1 \mathrm{e} \mathrm{bohr}^{-3}\right)$ and $\nabla^{2} \rho_{\mathrm{b}}$ exhibits small positive values $\left(<0.1 \mathrm{e} \mathrm{bohr}^{-5}\right)$. From the QTAIM point of view, the At- $\mathrm{C}_{1}$ bond falls in the regular closed-shell category [26], i.e. in the family of polar-covalent bond. The polarity index $p_{\text {AtC1 }}$ is always positive whatever the considered species (see Table 2). Thus, the orientation of the polarization vector is always directed from At to the bonded species in agreement with the weak electronegativity of At (recently calculated to 2.1 [42]). The atomic charge of At is always positive (see Table 3). It remains lower than $0.15 \mathrm{e}$, except for the At- $\mathrm{C} \equiv \mathrm{CH}$ species for which it reaches the value of $0.40 \mathrm{e}$, consistently with a high bond polarity index of 0.59 .

At this stage, the fundamental question of the bond weakening by SOC deserves a special attention. The Table 4 presents the effects of SOC on the ELF populations and QTAIM descriptors. 
Table 4. SOC effects on ELF populations and QTAIM descriptors.

\begin{tabular}{|c|c|c|c|c|c|c|c|c|}
\hline$\Delta S O^{a}$ & $\bar{C} C(A t)+V(A t)$ & \multicolumn{4}{|c|}{$\bar{V} V\left(A t, C_{l}\right)$} & \multicolumn{3}{|c|}{$\overline{A A t-C_{I}}$} \\
\hline System & 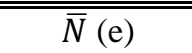 & 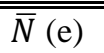 & $\overline{\overline{p_{A t C l}}}$ & 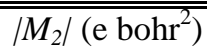 & $\overline{\pi^{6}}$ & 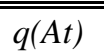 & 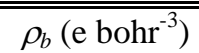 & $\bar{V}^{2} \rho_{b}\left(\mathrm{e}\right.$ bohr $\left.{ }^{-5}\right)$ \\
\hline 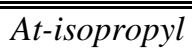 & 0.00 & "-0.01 & +0.03 & -0.09 & 0.00 & 0.00 & -0.007 & +0.015 \\
\hline At-vinyl & -0.01 & +0.04 & +0.02 & -0.01 & +0.05 & 0.00 & -0.007 & +0.015 \\
\hline At-phenyl & -0.01 & +0.01 & +0.02 & -0.01 & +0.03 & 0.00 & -0.007 & +0.015 \\
\hline At-ethynyl & +0.03 & +0.02 & +0.04 & -0.10 & +0.04 & +0.01 & -0.011 & +0.005 \\
\hline
\end{tabular}

${ }^{\mathrm{a}} \Delta \mathrm{SO}$ is defined as the difference between quasirelativistic and scalar-relativistic computed values.

${ }^{\mathrm{b}}$ The $\pi$ population (e) of valence basin $\mathrm{V}\left(\mathrm{At}, \mathrm{C}_{1}\right)$ is evaluated by only taking into account the expansion coefficients of the $\mathrm{p}_{\mathrm{x}}$ and $\mathrm{p}_{\mathrm{y}}$ Gaussian basis functions during the integration of the electron density over the basin volumes.

SOC effects (noted $\Delta \mathrm{SO}$ ) are here defined as the difference between quasirelativistic (2c-B3LYP) and scalar-relativistic (B3LYP) computed values. These results lead us to the non-ambiguous conclusion that SOC hardly affected the electronic structure. For example, the population of the ELF basin V(At, $\left.\mathrm{C}_{1}\right)$ are only changed within $0.04 \mathrm{e}$. The most affected descriptor by SOC is the $\nabla^{2} \rho_{\mathrm{b}}$ one for the At- $\mathrm{C}_{1}$ bond. Its value is decreased for all the studied species, meaning that the electrons are less stabilized close to the BCP. Note for the At- $\mathrm{CH}\left(\mathrm{CH}_{3}\right)_{2}$ species that $\nabla^{2} \rho_{\mathrm{b}}$ is so low that the SOC effect may disrupt the standard QTAIM classification: the At- $\mathrm{C}_{1}$ bond quits eventually the shared-shell category $\left(\nabla^{2} \rho_{\mathrm{b}}=-0.002 \mathrm{e}\right.$ bohr $\left.{ }^{-5}\right)$ to closed-shell one $\left(\nabla^{2} \rho_{\mathrm{b}}=0.013 \mathrm{e} \mathrm{bohr}^{-5}\right)$.

\section{Conclusion}

Using an approach that combines 2c-DFT calculations and topological analyses of (i) the electron density and (ii) the ELF function, we show that our methodology can provide new insights into the nature of the At-C bonds. It was evidenced that the polarcovalent At-C bond acquires some multiple bond character when At is bonded to a vinyl or a phenyl group instead of the isopropyl, and the bond multiplicity is even greater with an ethynyl group. The At-C bond strengths grow up accordingly. This finding may be of interest regarding the development of efficient ${ }^{211}$ At-labelling protocols for the alpha-radioimmunotherapy: better in vivo stability can be expected for the stronger At-C bonds. However, the At-C bond becomes more polarized as its strength is increased. In the case of the At- $\mathrm{C} \equiv \mathrm{CH}$ species, the At atom acquires a large positive charge, $+0.40 \mathrm{e}$, 
and one may think that ${ }^{211}$ At bonded to an sp carbon atom should be to much sensitive to oxidizing, enzymatic process, or more easily displaced by in vivo nucleophiles such as chloride, free amines, or thiolates. The At-C bond displays a relatively strong covalent bonding in the At- $\mathrm{C}_{6} \mathrm{H}_{5}$ species whilst retaining a small At positive charge $(+0.15 \mathrm{e})$. Hence, the presented results suggest that the aromatic carbon-astatine bond appears the best suited for ${ }^{211}$ At-labelling, in agreement with the previous empirical findings $[13,43]$.

\section{Acknowledgments}

The authors would like to dedicate this article to Prof Andreas Savin on the occasion of his 65 th birthday.

\section{Funding}

This work was supported by grants funded by the French National Agency for Research (ANR-2010-BLAN-0807), with "Investissements d'Avenir" (ANR-11-EQPX-0004, ANR-11-LABX-0018). It was carried out using HPC resources from GENCICINES/IDRIS (grant 2015-c2015085117) and CCIPL ("Centre de Calcul Intensif des Pays de la Loire").

\section{References}

[1] L. Visscher and K. G. Dyall, J. Chem. Phys. 104 (22), 9040-9046 (1996).

[2] T. Saue, K. Faegri and O. Gropen, Chem. Phys. Lett. 263 (3-4), 360-366 (1996).

[3] L. Visscher, J. Styszynski and W. C. Nieuwpoort, J. Chem. Phys. 105 (5), 19871994 (1996).

[4] R. F. W. Bader, Atoms in Molecules: A Quantum Theory. (Oxford University Press, New York, USA, 1994).

[5] B. Silvi and A. Savin, Nature 371 (6499), 683-686 (1994).

[6] B. Silvi, R. J. Gillespie and C. Gatti, in Comprehensive Inorganic Chemistry II (Second Edition), edited by J. R. Poeppelmeier (Elsevier, Amsterdam, 2013), pp. 187226.Amsterdam

[7] R. F. W. Bader, Chem. Rev. 91 (5), 893-928 (1991).

[8] B. Silvi, J. Pilme, F. Fuster and M. E. Alikhani, in Metal-Ligand Interactions, edited by N. Russo, D. Salahub and M. Witko (Springer Netherlands, 2003), Vol. 116, pp. 241-284

[9] X. Fradera, M. A. Austen and R. F. W. Bader, J. Phys. Chem. A 103 (2), 304314 (1998).

[10] R. J. Gillespie, E. A. Robinson and J. Pilmé, Chem. Eur. J. 16 (12), 3663-3675 (2010).

[11] J. Pilmé, E. Renault, T. Ayed, G. Montavon and N. Galland, J. Chem. Theory Comput. 8 (9), 2985-2990 (2012). 
[12] J. Pilmé, E. Renault, F. Bassal, M. Amaouch, G. Montavon and N. Galland, J. Chem. Theory Comput. 10 (11), 4830-4841 (2014).

[13] D. S. Wilbur, Curr Radiopharm. 1 (3), 144-176 (2008).

[14] L. Bo-Li, J. Yu-Tai, L. Zheng-Hao, L. Cheng, K. Masaharu and M. Minoru, Int. J. Appl. Radiat. Isot. 36 (7), 561-563 (1985).

[15] K. K. R. Pillai, W. W. McLaughlin, R. R. Lambrecht and W. W. Bloomer, J. Labelled Compd. Radiopharm. 24 (9), 1117-1122 (1987).

[16] G. W. M. Visser, E. L. Diemer and F. M. Kaspersen, Int. J. Appl. Radiat. Isot. 30 (12), 749-752 (1979).

[17] A. D. Becke and K. E. Edgecombe, J. Chem. Phys. 92 (9), 5397-5403 (1990).

[18] A. Savin, O. Jepsen, J. Flad, O. K. Andersen, H. Preuss and H. G. von Schnering, Angew. Chem. Int. 31 (2), 187-188 (1992).

[19] A. Savin, R. Nesper, S. Wengert and T. F. Fässler, Angew. Chem., Int. Ed. Engl. 36 (17), 1808-1832 (1997).

[20] C. Von Weizsacker, Zeitschrift für Physik 96 (7), 431-458 (1935).

[21] E. Matito, B. Silvi, M. Duran and M. Solà, J. Chem. Phys. 125 (2), 024301 (2006).

[22] R. H. Abraham, Shaw, C. D. , Dynamics: The Geometry of Behavior, C. Addison Wesley: Redwood City. (1992).

[23] R. H. M. Abraham, J. E. Foundations of Mechanics, C. Addison Wesley: Redwood City. (1994).

[24] R. F. W. Bader and M. E. Stephens, J. Am. Chem. Soc. 97 (26), 7391-7399 (1975).

[25] R. F. W. Bader and H. Essén, J. Chem. Phys. 80 (5), 1943-1960 (1984).

[26] W. Nakanishi and S. Hayashi, J. Phys. Chem. A 117 (8), 1795-1803 (2013).

[27] D. Cremer and E. Kraka, Angew. Chem., Int. Ed. Engl. 23 (8), 627-628 (1984).

[28] B. Silvi, J. Mol. Struct. 614 (1-3), 3-10 (2002).

[29] J. Pilmé and J.-P. Piquemal, J. Comput. Chem. 29 (9), 1440-1449 (2008).

[30] S. Raub and G. Jansen, Theor. Chem. Acc. 106 (3), 223-232 (2001).

[31] T. P. Straatsma, E. Aprà, T. L. Windus, E. J. Bylaska, W. de Jong, S. Hirata, et al., NWChem, A Computational Chemistry Package for Parallel Computers, version 5.1.1, Pacific Northwest National Laboratory, Richland, Washington (2008).

[32] P. J. Stephens, F. J. Devlin, C. F. Chabalowski and M. J. Frisch, J. Phys. Chem. 98 (45), 11623-11627 (1994).

[33] J. Champion, M. Seydou, A. Sabatie-Gogova, E. Renault, G. Montavon and N. Galland, Phys. Chem. Chem. Phys. 13 (33), 14984-14992 (2011).

[34] J. Champion, A. Sabatié-Gogova, F. Bassal, T. Ayed, C. Alliot, N. Galland, et al., J. Phys. Chem. A 117 (9), 1983-1990 (2013).

[35] J. Champion, C. Alliot, E. Renault, B. M. Mokili, M. Chérel, N. Galland, et al., J. Phys. Chem. A 114 (1), 576-582 (2010).

[36] K. A. Peterson, D. Figgen, E. Goll, H. Stoll and M. Dolg, J. Chem. Phys. 119 (21), 11113-11123 (2003).

[37] F. Weigend and A. Baldes, J. Chem. Phys. 133, 174102 (2010).

[38] D. Rappoport and F. Furche, J. Chem. Phys. 133 (13), 134105 (2010).

[39] S. Noury, X. Krokidis, F. Fuster and B. Silvi, Comput. Chem. 23 (6), 597-604 (1999).

[40] P. Flukiger, H. P. Luthi, S. Portmann and J. Weber, Molekel, version 4.3, Swiss Center for Scientific Computing, Manno, Switzerland (2002).

[41] L. Vasaros, Y. V. Norseev and V. A. Khalkin, Dokl. Akad. Nauk SSSR 263, 119-123 (1982). 
[42] J. Furtado, F. De Proft and P. Geerlings, J. Phys. Chem. A 119 (8), 1339-1346 (2015).

[43] G. Vaidyanathan and M. R. Zalutsky, Curr Radiopharm. 4 (4), 283-294 (2011). 